\title{
Gut Microbiome-Derived Uremic Toxin Levels in Hemodialysis Patients on Different Phosphate Binder Therapies
}

\author{
Lin-Chun Wang $^{\mathrm{a}}$ Leticia M. Tapia $^{\mathrm{a}}$ Xia Tao $^{\mathrm{a}}$ Joshua E. Chao ${ }^{\mathrm{a}}$ Ohnmar Thwin $^{\mathrm{a}}$ \\ Hanjie Zhang $^{\text {a }}$ Stephan Thijssen $^{\text {a }}$ Peter Kotanko $^{\text {a, b }}$ Nadja Grobe ${ }^{a}$ \\ aRenal Research Institute, New York, NY, USA; blcahn School of Medicine at Mount Sinai, New York, NY, USA
}

\section{Keywords}

Hemodialysis · Uremic toxins · Phosphate binders .

Sucroferric oxyhydroxide $\cdot$ Sevelamer carbonate

\begin{abstract}
Introduction: Constipation is prevalent in patients with kidney failure partly due to the use of medication, such as phosphate binders. We hypothesized that serum levels of gut microbiome-derived uremic toxins (UTOX) may be affected by the choice of phosphate binder putatively through its impact on colonic transit time. We investigated two commonly prescribed phosphate binders, sevelamer carbonate (SEV) and sucroferric oxyhydroxide (SFO), and their association with gut microbiome-derived UTOX levels in hemodialysis (HD) patients. Methods: Weekly blood samples were collected from 16 anuric HD participants during the 5 -week observational period. All participants were on active phosphate binder monotherapy with either SFO or SEV for at least 4 weeks prior to enrollment. Eight UTOX (7 gut microbiomederived) and tryptophan were quantified using liquid chromatography-mass spectrometry. Serum phosphorus, nutritional, and liver function markers were also measured. For each substance, weekly individual levels, the median concentration per participant, and differences between SFO and SEV groups were reported. Patient-reported bowel move-
\end{abstract}

ments, by the Bristol Stool Scale (BSS), and pill usage were assessed weekly. Results: The SEV group reported a 3.3-fold higher frequency of BSS stool types 1 and 2 (more likely constipated, $p<0.05$ ), whereas the SFO group reported a 1.5fold higher frequency of BSS stool types 5-7 (more likely loose stool and diarrhea, not significant). Participants in the SFO group showed a trend toward better adherence to phosphate binder therapy (SFO: $87.6 \%$ vs. SEV: $66.6 \%$, not significant). UTOX, serum phosphorus, nutritional and liver function markers, and tryptophan were not different between the two groups. Conclusion: There was no difference in the gut microbiome-derived UTOX levels between phosphate binders (SFO vs. SEV), despite SFO therapy resulting in fewer constipated participants. This pilot study may inform study design of future clinical trials and highlights the importance of including factors beyond bowel habits and their association with UTOX levels.

(c) 2021 The Author(s)

Published by S. Karger AG, Basel

\section{Introduction}

The loss of kidney function in kidney failure patients is accompanied by the retention of a host of metabolites [1]. Many of these uremic retention solutes have been shown to negatively affect the functioning of cells and or- karger@karger.com www.karger.com/bpu

Karger $\stackrel{\text { ' }}{5}$

bOPEN ACCESS
(C) 2021 The Author(s)

Published by S. Karger AG, Basel

This is an Open Access article licensed under the Creative Commons Attribution-NonCommercial-4.0 International License (CC BY-NC) (http://www.karger.com/Services/OpenAccessLicense), applicable to the online version of the article only. Usage and distribution for commercial purposes requires written permission.
Correspondence to:

Nadja Grobe, nadja.grobe@ rriny.com 
gans, resulting in the uremic syndrome [2]. Some of these uremic toxins (UTOX) are derived from colon microbes [3-7]. Gut microbiome dysbiosis - changes in composition and function of the gut microbiome - is common in kidney failure patients. One contributing factor to gut dysbiosis is the change of gastrointestinal tract environment by the influx of urea, uric acid, and oxalate from the blood into the gut. A previous study revealed that the dominant microbiota in kidney failure patients are families expressing urease, urate oxidase, and $p$-cresol- and indole-forming enzymes [8]. These enzymes generate metabolites that are subsequently absorbed into the blood and, in some cases, further metabolized into UTOX in the liver. Some toxins are highly protein-bound and thus are inefficiently removed by conventional hemodialysis (HD) treatment [9]. Their accumulation in kidney failure patients may contribute to systemic inflammation and chronic kidney disease (CKD) progression [10-12]. Further, elevated levels of these protein-bound UTOX have been associated with higher all-cause and cardiovascular mortality [13]. However, it is important to acknowledge that to date no causal relationship between colon-derived UTOX and poor clinical outcomes has been established.

Dialysis patients frequently suffer from constipation as a result of prolonged colonic transit time [14]. Prolonged colonic transit time can result in increased protein fermentation, altered gut microbiota, and subsequent changes in the production of UTOX precursors [7]. Chronic constipation is partly due to the use of medication, such as phosphate binders [14]. Interestingly, one of the significant side effects of iron-based (sucroferric oxyhydroxide [SFO]) phosphate binders is more frequent bowel movements, loose stool, and diarrhea [15-17]. If patients on SFO (Velphoro ${ }^{\circledR}$ ) experience more frequent bowel movements, their colonic transit time may be shorter than patients on a widely prescribed phosphate binder, sevelamer carbonate (SEV; Renvela $\left.{ }^{\circledR}\right)$, who reported higher rates of constipation [15]. The combination of these factors may induce overgrowth of proteolytic species, which favors the production of both toxic metabolites (e.g., p-cresyl sulfate [PCS], phenyl acetic acid, indoxyl sulfate [IS], and indole 3 -acetic acid) and proinflammatory substances (e.g., PCS) in kidney failure patients $[18,19]$.

We hypothesized that serum levels of gut microbiomederived UTOX may be affected by the choice of phosphate binder putatively through its impact on colonic transit time. This hypothesis was built on two observations: (a) clinically, SEV induces constipation, while SFO causes transient diarrhea [15]; (b) colonic transit time im- pacts the generation of gut-derived UTOX as longer colonic transit time is associated with higher UTOX levels [7].

\section{Materials and Methods}

Study Design

The study presented here is a multicenter, 6-week observational study conducted at $4 \mathrm{HD}$ clinics in New York, NY, USA. The inclusion criteria were kidney failure patients on thrice weekly HD regimen, $\geq 18$ years of age at the time of informed consent, and on active phosphate binder monotherapy with either SFO or SEV for at least 4 weeks prior to enrollment. Seventeen study participants were enrolled from September 2018 to March 2019 with no followup afterward. The exclusion criterion was inability to maintain diary to track binder usage and bowel movements. Sixteen study participants completed all study visits; 1 patient was withdrawn due to relocation (Fig. 1). All study participants were anuric and treated with polysulfone dialyzers.

Study participants were seen on a scheduled mid-week treatment day once a week for 6 consecutive weeks. An interview was administered in the first week, which included study participants' binder prescription and bowel habits. In the subsequent 5 weeks, predialysis blood samples were collected to measure UTOX and other biomarkers related to phosphate binder therapy, nutritional status, and liver function. Seven gut-derived UTOX were selected to test the hypothesis: trimethylamine-N-oxide (TMAO), indoxyl glucuronide (IG), phenylacetylglutamine (PAG), IS, 3-carboxy-4-methyl-5-propyl2-furanpropionic acid (CMPF), PCS, and hippuric acid (HA; derived from both the gut microbiome and mammalian sources). For comparison, we also measured mammalian-derived kynurenic acid (KYNA) and nutritional marker tryptophan (TRP). A weekly diary was provided to the study participants to capture phosphate binder usage and bowel movements. Satisfaction and frequency of bowel movements along with stool consistency, determined by Bristol Stool Scale (BSS) [20], were assessed to indicate the bowel habit for each patient. On the BSS, types 1 and 2 were considered as likely constipated, types 5-7 as likely loose stool or diarrhea, and types 3 and 4 were regarded as normal. Bowel satisfaction was evaluated using a numeric scale from 1 (unsatisfactory) to 10 (completely satisfactory). The adherence to phosphate binder therapy was determined by weekly pill counts. The adherence was calculated as the percentage of the actual number (pill count) to the expected number (prescription) of pills consumed. UTOX levels, phosphate binder usage and bowel habits, were analyzed until March 2021. All study procedures were approved by Western Institutional Review Board ${ }^{\circledR}$ (Protocol number: \#20181972) and performed in accordance with the principles of the Declaration of Helsinki. The signed written informed consent forms were obtained from all participants for this study.

\section{Sample Preparation}

Serum was aliquoted and stored at $-80^{\circ} \mathrm{C}$ until analysis. For UTOX and TRP analysis, samples were prepared according to Dunn et al. [21] with some modifications. Briefly, $50 \mu \mathrm{L}$ serum was spiked with $25 \mu \mathrm{L}$ internal standard cocktail consisting of $\left[\mathrm{D}_{9}\right]$ trimethylamine $\mathrm{N}$-oxide, $\left[{ }^{13} \mathrm{C}_{6}\right]$-IS, and $\left[\mathrm{D}_{7}\right]$-PCS (Cambridge Isotope Laboratories, Inc., Tewksbury, MA, USA). Aliquots of serum from each participant were combined and served as quality control 


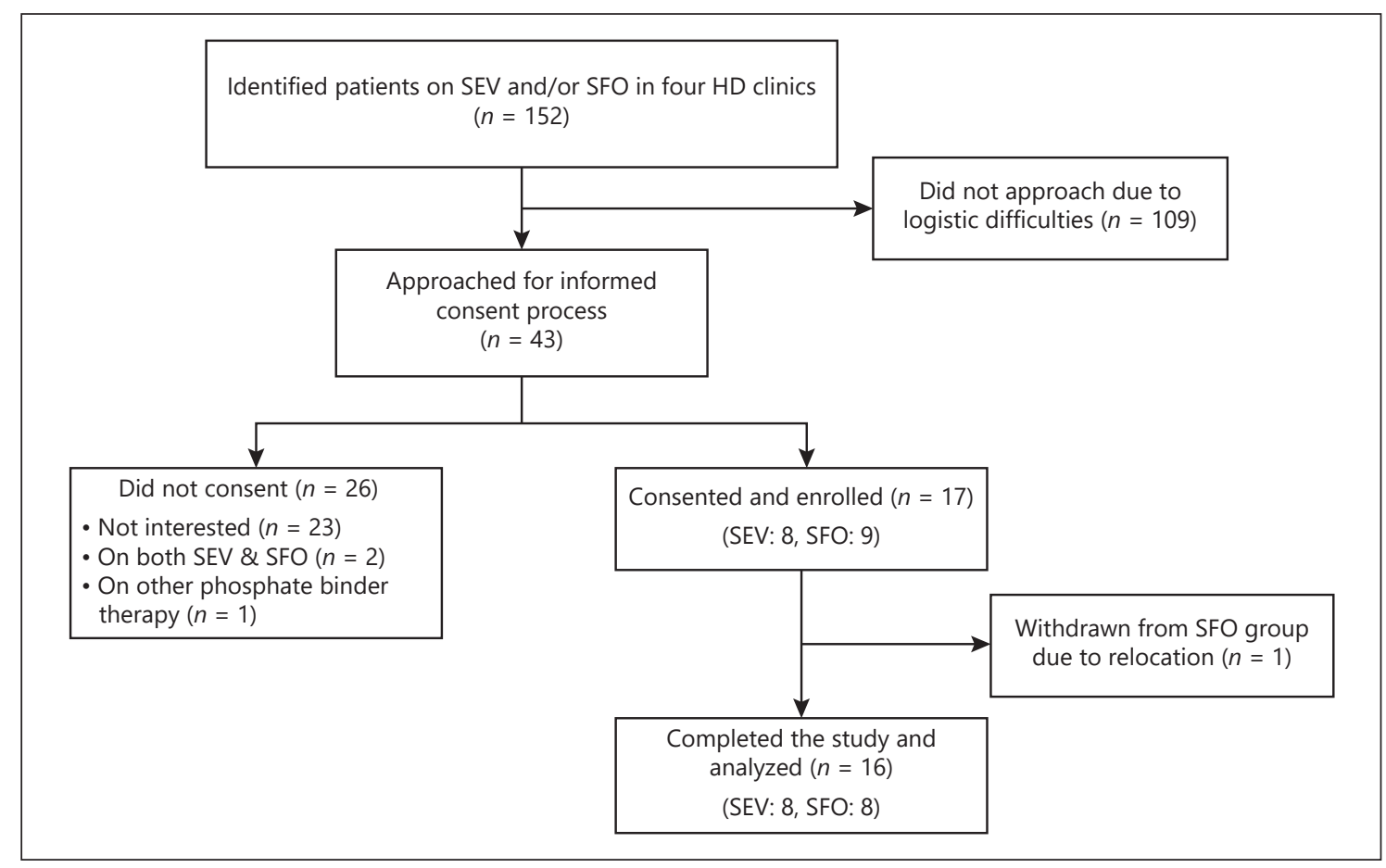

Fig. 1. Flowchart of the cohort. SEV, sevelamer; SFO, sucroferric oxyhydroxide; HD, hemodialysis.

samples. An external standard curve for each substance of interest quantified by liquid chromatography-mass spectrometry (LC-MS) was prepared in $4 \%$ human serum albumin. Samples were extracted with $150 \mu \mathrm{L}$ ice-cold methanol followed by centrifugation for $15 \mathrm{~min}$ at $4,300 \mathrm{rpm}$ and $4^{\circ} \mathrm{C}$. The supernatant was dried down using a centrifugal vacuum evaporator without heating and dried extracts were resuspended in $0.01 \%$ formic acid and $1 \mathrm{mM}$ ammonium formate for LC-MS analysis.

\section{Sample Analysis}

Serum phosphate, nutritional markers, and liver enzymes were analyzed using a Pentra C400 biochemistry analyzer (Horiba, Irvine, CA). UTOX and TRP levels were analyzed using a 1290 Infinity II high-performance liquid chromatograph coupled to a 6545 Quadrupole Time-of-Flight mass spectrometer (Agilent Technologies Inc., Santa Clara, CA, USA). UTOX and TRP were separated on a reverse-phase column (Luna C18 $4.6 \times 150 \mathrm{~mm}, 3 \mu \mathrm{m}$, Phenomenex, Torrance, CA, USA) at a flow rate of $0.75 \mathrm{~mL} / \mathrm{min}$. The gradient mobile phase consisted of mobile phase A $(0.01 \%$ formic acid and $1 \mathrm{~mm}$ ammonium formate in water) and mobile phase $\mathrm{B}$ ( $0.01 \%$ formic acid and $1 \mathrm{mM}$ ammonium formate in methanol). The gradient started at $1 \%$ mobile phase $\mathrm{B}$, which was changed linearly over the next 6.5 min until reaching $40 \%$. Over the next 1.5 $\mathrm{min}$, the percentage was increased linearly to $90 \%$ followed by a linear increase to $100 \%$ over $3.5 \mathrm{~min}$, where it was kept for $0.5 \mathrm{~min}$. The mobile-phase composition was allowed to return to the initial conditions, which were held for $3 \mathrm{~min}$ to equilibrate the column. The following MS parameters were used: $m / z 50-1,000$, gas temperature $350^{\circ} \mathrm{C}$, drying gas $12 \mathrm{~L} / \mathrm{min}$, nebulizer $35 \mathrm{psig}$, sheath gas temperature $400^{\circ} \mathrm{C}$, sheath gas flow $12 \mathrm{~L} / \mathrm{min}$, fragmentor $125 \mathrm{~V}$ and $175 \mathrm{~V}$, skimmer $65 \mathrm{~V}$, Oct 1 RF Vpp $750 \mathrm{~V}$, VCap 4,000 V, and Nozzle Voltage $500 \mathrm{~V}$ for positive mode; VCap 2,000 V and Nozzle Voltage 2,000 V for negative mode. A standard curve consisting of 5 levels for UTOX and TRP was used to calculate accuracy and precision for 3 quality control samples measured in triplicates at low, mid, and high concentrations of UTOX and TRP.

\section{Statistical Analysis}

Descriptive statistics including measures of central tendency (mean, median) and spread (standard deviation, interquartile range) for continuous variables and number and frequency for categorical variables were compiled. For each UTOX, TRP, and routine clinical diagnostic biomarkers, we calculated the median concentration per participant and then the median across all participants within each group. We also reported the differences in median serum concentrations between the treatment groups. A mixed-effects model was employed to investigate UTOX levels between different binder therapies (SEV vs. SFO) during the observational period (R Foundation for Statistical Computing, Vienna, Austria).

\section{Results}

There was no significant difference between the two treatment groups regarding sex, age, race, body mass index, comorbidities, dialysis vintage, adherence to binder therapy, duration of phosphate binder treatment, Kt/V, and normalized protein catabolic rate (Table 1). Pill bur- 
Table 1. Study participants' characteristics

\begin{tabular}{lccc}
\hline Characteristic & Total $(n=16)$ & SEV $(n=8)$ & SFO $(n=8)$ \\
\hline Female/male & $5 / 11$ & $3 / 5$ & $2 / 6$ \\
Age, years & $55.1 \pm 13.7$ & $58.3 \pm 13.5$ & $51.9 \pm 14.0$ \\
BMI, kg/m ${ }^{2}$ & $27.5 \pm 5.9$ & $27.1 \pm 7.1$ & $27.9 \pm 4.9$ \\
Hemodialysis vintage, years & $4.8 \pm 3.8$ & $5.1 \pm 4.6$ & $4.4 \pm 3.2$ \\
Equilibrated Kt/V & $1.38 \pm 0.16$ & $1.42 \pm 0.20$ & $1.35 \pm 0.12$ \\
Duration of phosphate binder treatment, years & $2.0 \pm 1.6$ & $3.0 \pm 1.9$ & $1.3 \pm 0.8$ \\
Phosphate binder treatment adherence, $\%$ & $77.1 \pm 34.5$ & $66.6 \pm 25.0$ & $87.6 \pm 40.9$ \\
Bowel habit satisfaction score** & $6.3 \pm 2.6$ & $5.5 \pm 3.5$ & $7.1 \pm 1.5$ \\
nPCR, g/kg/day & $1.03 \pm 0.17$ & $1.05 \pm 0.22$ & $1.01 \pm 0.12$ \\
Race, $n$ (\%) & & & $4(50)$ \\
White & $8(50)$ & $4(50)$ & $3(37)$ \\
African-American & $7(44)$ & $4(50)$ & $1(13)$ \\
Asian & $1(6)$ & $0(0)$ & $2(25)$ \\
Comorbidities, $n$ (\%) & & & $7(88)$ \\
Type 2 diabetes & $3(19)$ & $1(13)$ & $0(0)$ \\
Hypertension & $13(81)$ & $6(75)$ & $8(100)$ \\
Chronic heart failure & $1(6)$ & $1(13)$ & $2(25)$ \\
Secondary hyperparathyroidism & $16(100)$ & $8(100)$ & $1(13)$ \\
HIV & $3(19)$ & & \\
\hline
\end{tabular}

Values are mean \pm standard deviation or count (percentage) as appropriate. BMI, body mass index; SEV, sevelamer carbonate; SFO, sucroferric oxyhydroxide; nPCR, normalized protein catabolic rate; HIV, human immunodeficiency virus.

* The phosphate binder treatment adherence was calculated as the percentage of the actual number (pill count) to the expected number (prescription) of pills consumed.

Adherence $=\frac{\text { pills taken }(\text { pill count })}{\text { perscription }(\text { pill number expected to be taken })} \times 100 \%$.

** Bowel habit satisfaction on a scale of 1-10 was assessed at the time of enrollment and reported by the study participants.

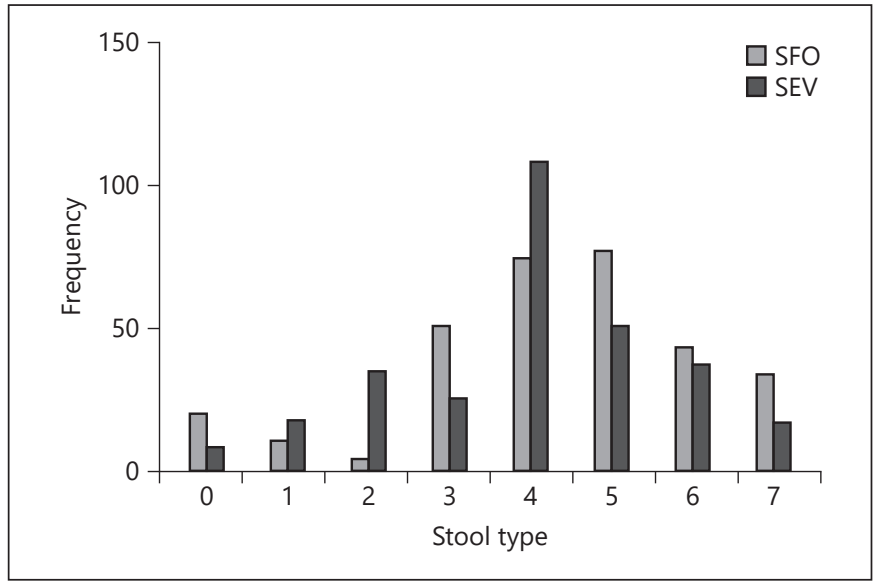

Fig. 2. Bowel movements in 16 participants over 5 weeks stratified per treatment group. Stool types 1-7 were categorized according to the Bristol Stool Scale. The number 0 is not part of the Bristol Stool Scale. It means an absence of a daily bowel movement. Type 1 , nut-like; type 2, lumpy sausage; type 3 , sausage with cracks; type 4 , smooth snake; type 5 , soft blobs; type 6 , fluffy pieces; type 7 , watery. SFO, sucroferric oxyhydroxide; SEV, sevelamer carbonate. den was lower in the SFO group versus the SEV group (mean 2.6 vs. 6.4, respectively). Simultaneous lanthanum carbonate intake was recorded for 1 patient in the SEV group. The SEV group reported a 3.3-fold higher frequency of stool types 1 and 2 (more likely constipated, $p<0.05$ ), whereas the SFO group reported a trend toward a 1.5-fold higher frequency of stool types 5-7 (more likely loose stool and diarrhea, not significant) (Fig. 2). When scoring bowel habit satisfaction on a scale from 1 (unsatisfactory) to 10 (completely satisfactory), patients in the SFO group showed a trend toward higher scores than the SEV group (7.1 vs. 5.5, respectively). Individual phosphate levels, nutritional markers, such as normalized protein catabolic rate, albumin and total protein, liver function markers, and total and direct bilirubin were assessed over 5 weeks (Fig. 3; Table 1). Phosphate levels did not differ between the SEV and SFO groups (Table 2). Nutritional markers, such as albumin and total protein, liver function markers, and total and direct bilirubin were not different between the 2 groups (Table 2). A total 


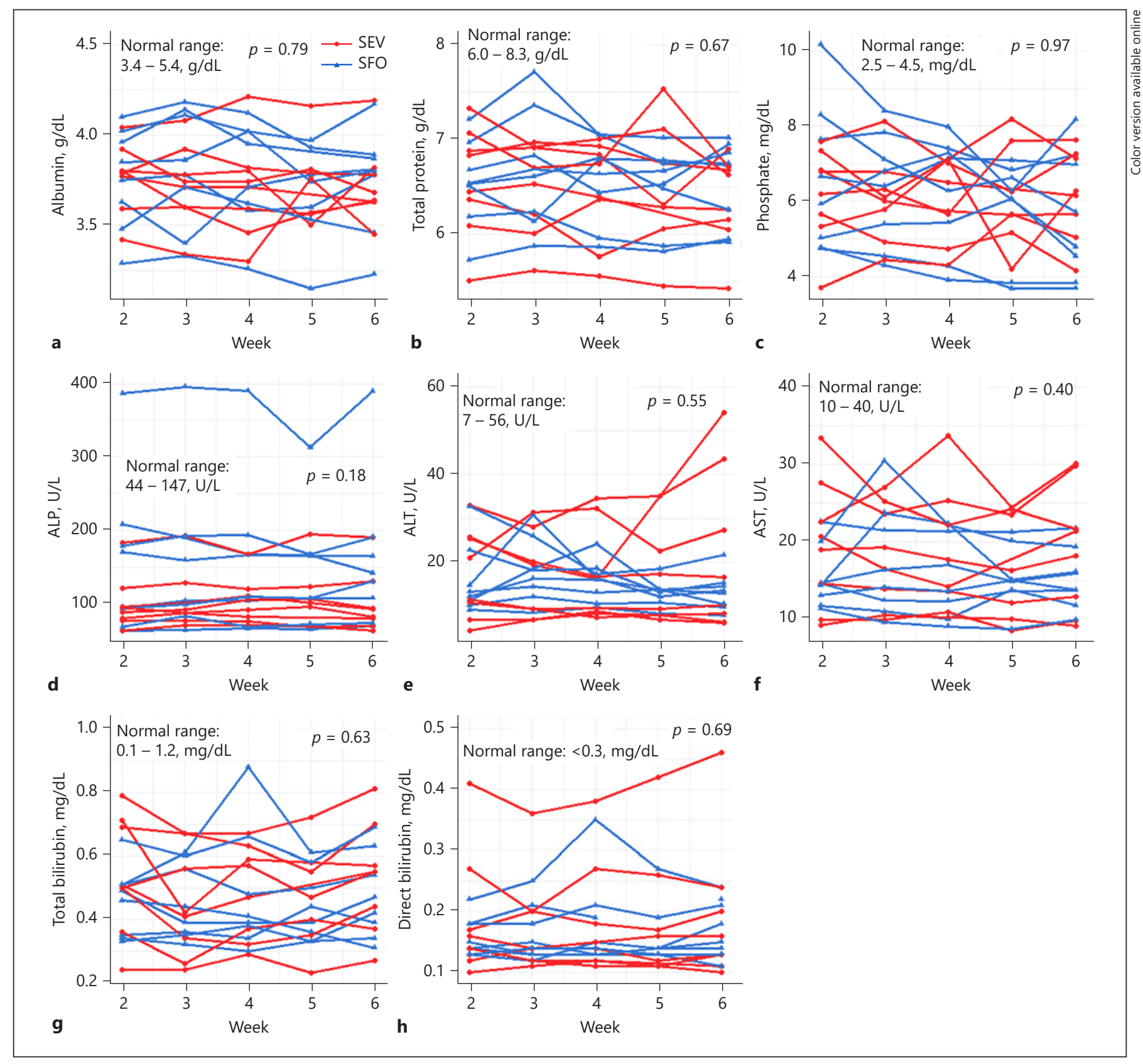

Fig. 3. Serum levels of albumin (a), total protein (b), phosphate (c), $\operatorname{ALP}(\mathbf{d}), \operatorname{ALT}(\mathbf{e}), \operatorname{AST}(\mathbf{f})$, total bilirubin (g), and direct bilirubin (h) in 16 study participants over 5 weeks stratified per treatment group. SFO, sucroferric oxyhydroxide; SEV, sevelamer carbonate.

of 9 substances were measured by LC-MS weekly over 5 weeks for each patient shown in Figure 4: 7 gut microbiome-derived UTOX (TMAO, IG, PAG, IS, CMPF, PCS, and HA), 1 mammalian-derived UTOX (KYNA), and 1 nutritional marker (TRP). UTOX and TRP levels did not differ between the SEV and SFO groups (Table 3; Fig. 4).
Comparison between groups over time was analyzed by a mixedeffects model. The threshold for statistical significance was set to $p$ $<0.05$. ALP, alkaline phosphatase; ALT, alanine aminotransferase; AST, aspartate aminotransferase. 


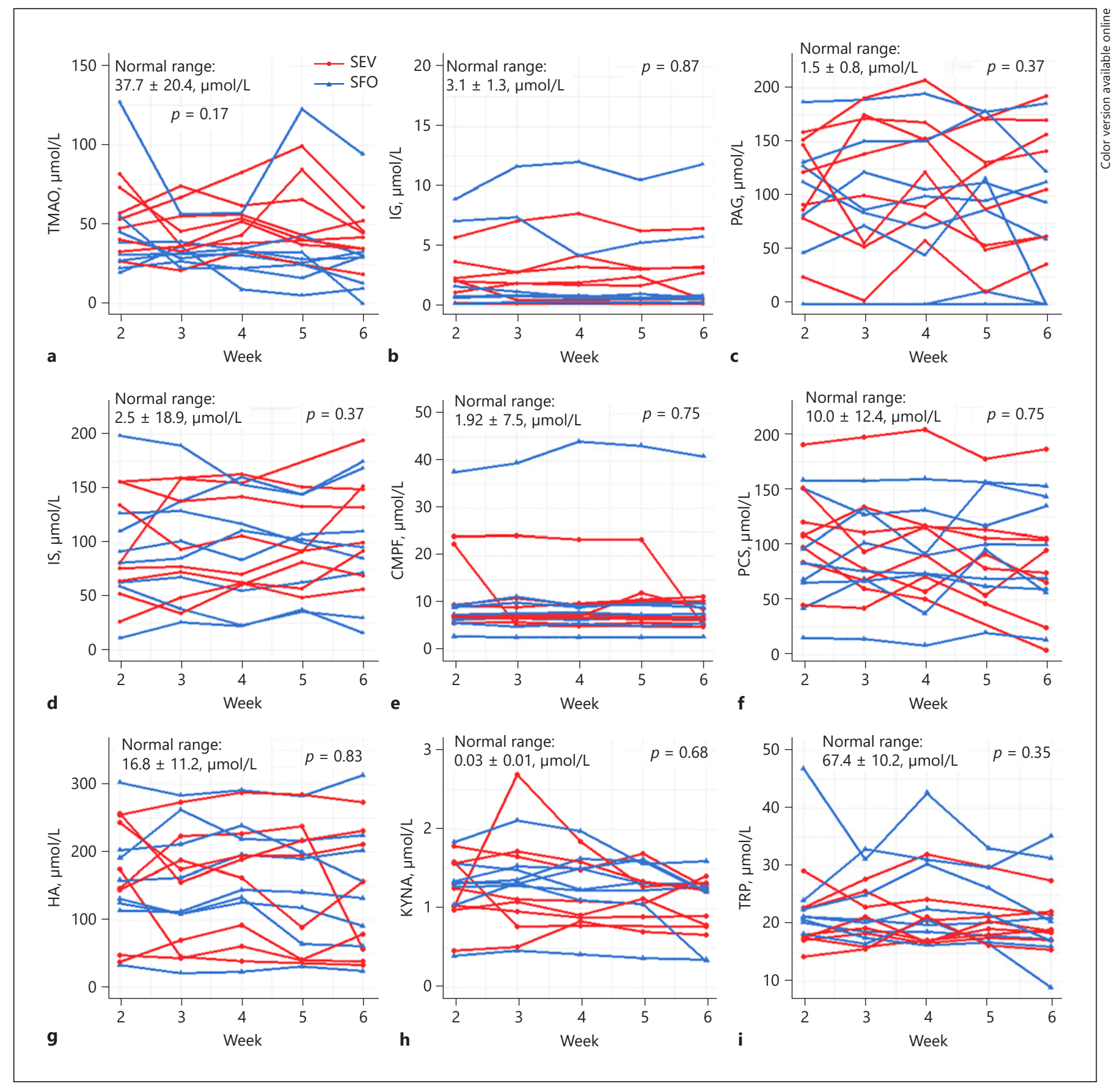

Fig. 4. Serum levels of UTOX and tryptophan in 16 participants over 5 weeks stratified per treatment group. TMAO (a), IG (b), PAG (c), IS (d), CMPF (e), PCS (f), HA (g), KYNA (h), and TRP (i). SFO, sucroferric oxyhydroxide; SEV, sevelamer carbonate; TMAO, trimethylamine-N-oxide, IG, indoxyl glucuronide, PAG, phenylacetylglutamine; IS, indoxyl sulfate; CMPF, 3-carboxy-4-methyl-5-propyl-2-furanpropionic acid; PCS, $p$-cresyl sulfate; HA, hippuric acid; KYNA, kynurenic acid; TRP, tryptophan. Comparison between groups over time was analyzed by a mixed-effects model. The threshold for statistical significance was set to $p<0.05$. 
Table 2. Routine clinical diagnostic biomarkers related to nutritional status, phosphate binder therapy, and liver function

\begin{tabular}{lllllllrr}
\hline Substance & Albumin, g/dL & Total Pt, g/dL & Phosphate, mg/dL & ALP, U/L & ALT, U/L & AST, U/L & B-T, mg/dL & B-D, mg/dL \\
\hline Normal range & $3.4-5.4$ & $6.0-8.3$ & $2.5-4.5$ & $44-147$ & $7-56$ & $10-40$ & $0.1-1.2$ \\
SFO & $3.8(0.3)$ & $6.7(0.4)$ & $6.3(2.0)$ & $136.3(77.7)$ & $14.2(3.0)$ & $14.9(4.0)$ & $0.4(0.2)$ & $0.14(0.06)$ \\
SEV & $3.7(0.2)$ & $6.6(0.6)$ & $6.1(1.2)$ & $92.7(26.5)$ & $13.4(15.8)$ & $18.4(11.7)$ & $0.5(0.2)$ & $0.15(0.08)$ \\
$\Delta$ Median & 0.08 & 0.10 & 0.29 & 43.61 & 0.73 & -3.51 & -0.12 & -0.01 \\
$p$ value & 0.79 & 0.67 & 0.97 & 0.18 & 0.55 & 0.40 & 0.63 \\
\hline
\end{tabular}

Values are median (interquartile range $[\mathrm{IQR}]$ ) serum concentrations in each group. IQR was calculated as the difference between the third and the first quartiles. A mixed-effects model was used for a $p$ value analysis. SFO, sucroferric oxyhydroxide; SEV, sevelamer carbonate; $\Delta$ Median, the median value in the SFO group minus the median value in the SEV group; total Pt, total protein; ALP, alkaline phosphatase; ALT, alanine aminotransferase; AST, aspartate aminotransferase; B-T, total bilirubin; B-D, direct bilirubin.

Table 3. Serum uremic toxins and tryptophan levels

\begin{tabular}{|c|c|c|c|c|c|c|c|c|c|}
\hline \multirow[t]{2}{*}{ Substance } & \multicolumn{7}{|c|}{ Gut microbiome-derived } & \multirow{2}{*}{$\begin{array}{l}\text { Mammalian- } \\
\text { derived } \\
\text { KYNA }\end{array}$} & \multirow{2}{*}{$\begin{array}{l}\text { Nutritional } \\
\text { marker } \\
\text { TRP }\end{array}$} \\
\hline & TMAO & IG & PAG & IS & CMPF & PCS & $\mathrm{HA}^{*}$ & & \\
\hline Reference levels ${ }^{\mathrm{a}, \mathrm{b}}$ & $37.7 \pm 20.4$ & $3.1 \pm 1.3$ & $1.5 \pm 0.8$ & $2.5 \pm 18.9$ & $19.2 \pm 7.5$ & $10.0 \pm 12.4$ & $16.8 \pm 11.2$ & $0.03 \pm 0.01$ & $67.4 \pm 10.2$ \\
\hline $\mathrm{SFO}$ & $31.5(10.2)$ & $0.8(1.7)$ & $92.2(81.6)$ & $98.5(68.0)$ & $1.8(0.7)$ & $87.0(68.5)$ & $160.8(90.4)$ & $1.3(0.2)$ & $21.0(8.3)$ \\
\hline SEV & $39.8(22.5)$ & $1.9(2.7)$ & $111.6(97.0)$ & $88.8(79.1)$ & $1.8(0.8)$ & $88.8(37.4)$ & $175.2(154.4)$ & $1.0(0.5)$ & $18.6(5.0)$ \\
\hline$\Delta$ Median & -8.3 & -1.1 & -19.4 & 9.6 & 0.0 & -1.8 & -14.4 & 0.3 & 2.4 \\
\hline$p$ value & 0.17 & 0.87 & 0.37 & 0.37 & 0.75 & 0.75 & 0.83 & 0.68 & 0.35 \\
\hline
\end{tabular}

Reference levels are mean \pm standard deviation in $\mu$ mol/L. Median (interquartile range [IQR]) values of serum concentrations in each group in $\mu \mathrm{mol} / \mathrm{L}$ are shown. IQR was calculated as the difference between the third and the first quartiles. A mixed-effects model was used for a $p$ value analysis. SFO, sucroferric oxyhydroxide; SEV, sevelamer carbonate; $\triangle$ Median, the median value in the SFO group minus the median value in the SEV group; TMAO, trimethylamineN-oxide; IG, indoxyl glucuronide; PAG, phenylacetylglutamine; IS, indoxyl sulfate; CMPF, 3-carboxy-4-methyl-5-propyl-2-furanpropionic acid; PCS, p-cresyl sulfate; HA, hippuric acid; KYNA, kynurenic acid; TRP, tryptophan. ${ }^{*} \mathrm{HA}$ is derived from both the gut microbiome and mammalian source. ${ }^{a}$ Database.uremic-toxins.org/soluteList.php. ${ }^{\mathrm{b}}$ Geisler et al. Serum tryptophan, kynurenine, phenylalanine, tyrosine, and neopterin concentrations in 100 healthy blood donors. Pteridines 2015;26(1):31-36.

resulted in fewer constipated participants, and gut microbiome-derived UTOX levels were not different between the two groups. To the best of our knowledge, we are the first group to compare UTOX levels in HD patients on SFO or SEV in the same setting. Our pilot study could inform future studies, especially for power analysis and sample size estimation.

The bowel habits result from the present study is in agreement with Floege et al. [15], who reported a higher frequency of mild transient diarrhea in patients taking SFO compared to SEV ( $20 \%$ vs. $7 \%$, respectively) and a higher frequency of constipation with SEV compared to SFO ( $7.2 \%$ vs. $3.8 \%$, respectively). In a later phase III, open-label, long-term study to assess safety and efficacy of SFO, the frequency of diarrhea was similarly high at $22.4 \%$ [17]. The consistency and frequency of stool could be an indicator of prolonged colonic transit time. $\mathrm{Wu}$ et al. [22] reported that total, right segmental as well as rec- tosigmoid segmental colonic transit times were prolonged in HD patients compared with healthy controls [22]. They further observed that increased individual colonic transit times were correlated with self-reported constipation in HD patients.

The colon is the main site of gut-derived uremic solute production [23]. Prolonged colonic transit time, in addition to the altered composition and function of gut microflora, may result in longer time available for bacterial proliferation [7]. Long colonic transit time is accompanied by a shift in colonic metabolism from carbohydrate fermentation to protein catabolism as reflected by higher urinary levels of potentially deleterious protein-derived metabolites [24]. Delivery of undigested protein to the colon may generate more uremic toxin precursors (such as thiols, phenols, and indoles) through proteolytic fermentations and the toxic waste products are absorbed into systemic circulation [25]. Indeed, studies showed disturbed bowel habits, character- 
ized by slow transit time (constipation), which may lead to production of PCS $(p=0.02)$ in nondiabetic nondialysisdependent CKD patients [26] and higher levels of total and free PCS $(p<0.01)$ and total indole acetic acid $(p=0.04)$ in patients on automatized peritoneal dialysis [27]. While the impact of SEV and SFO on colonic transit time is well established $[14,15]$, we cannot exclude the possibility that inherent differences in the agents themselves may affect solute production or intestinal uptake. Our pilot study strongly suggests that it is crucial for future trials to include detailed information on microbiome composition, colonic metabolism, and dietary habits to understand the causal relationship between colonic transit time and individual uremic toxin levels.

Phosphate binders are also associated with gastrointestinal distress, and they may bind molecules other than phosphate and may alter the gut microbiota, altogether having systemic effects unrelated to phosphate control [28]. The impact of SEV on uremic toxin levels has been controversial. In a large-scale $(N=423)$ observational study by Dai et al. [29] comparing SEV users with nonusers, SEV use was associated with high serum IS. Another observational study found a significant reduction in the levels of PCS, not IS, after a 3-month period of SEV treatment [30]. The precursor of PCS, $p$-cresol, was found to be significantly reduced after SEV treatment in a singleblind, randomized placebo-controlled trial with patients with stage 3-5 CKD (not on dialysis) [31]. In contrast, Brandenburg et al. [32] reported that 8 weeks of sevelamer hydrochloride treatment increased serum PCS but did not change serum IS levels in HD patients. Furthermore, in a multicenter, double-blind, placebo-controlled randomized clinical trial to evaluate the adsorptive effect of SEV in stage $3 \mathrm{~b}-4 \mathrm{CKD}$ patients, there was no significant decrease in the serum concentration of PCS, IS, and indole acetic acid after a 12-week course of treatment [33]. Similarly, in a secondary analysis of the NICOREN trial, SEV did not reduce the circulating levels of proteinbound toxins in HD patients [34].

To date, the knowledge of the impact of SFO on UTOX levels is limited. Only one study explored the impact of SFO on UTOX in HD patients [35] and revealed that, despite a lack of change in the diversity and composition of the microbiome, UTOX (IS and PCS) were increased. It is important to note that the design of this and our study is different. Iguchi et al. studied HD patients before and after 3 months of SFO administration. In the present study, participants were already on SFO binder therapy for at least 4 weeks (on average $1.3 \pm 0.8$ years) and UTOX levels were measured over a shorter period of 5 weeks.
Out of the 9 substances detected by LC-MS in HD serum in the present study, TMAO, IG, PAG, IS, PCS, and $\mathrm{HA}$ undergo metabolism in the liver before entering the systemic blood stream. The participants in our study did not show any liver impairment, as three liver markers were not different between the two treatment groups. At the individual level, alkaline phosphatase (ALP) was increased in four SFO participants and one SEV participant. Since ALP exists in blood in numerous distinct forms, which originate mainly from bone and liver, slightly elevated ALP may indicate a disturbance in bone mineral metabolism [36], which is quite common in the kidney failure population. Consistent with previously published literature [37, 38], the two binders SEV and SFO demonstrated equivalent control of serum phosphorus. Additionally, we found that study participants in the SFO group tended to be more compliant to the medication (87.6\%) as opposed to patients in the SEV group (66.6\%); this may be a result from the lower pill burden of SFO [39].

The limitations of our study are its observational nature and a small sample size, which may mask the impacts of phosphate binder on UTOX blood levels. The evaluations of stool consistency and bowel movements rely primarily on patient-reported information. We observed discrepancies between patient-reported medication consumption and actual pill count. This observation raises a concern about the reliability of patient-reported data and, along with the small sample size, prevents us from conducting further analysis of adjusting confounding factors. Similar to previously published literature by Floege et al. [15], nonadherence was slightly more common in the SEV group than in the SFO group. Evaluation of fecal specimen may give more insightful information with regard to therapy adherence and gut microflora. However, due to logistical difficulties, fecal samples were not obtained during the study. Another drawback of our study is the lack of a detailed dietary assessment that would allow us to capture the protein-to-fiber ratio and the direct intake of UTOX precursors.

In conclusion, there was no difference in the serum gut microbiome-derived UTOX levels between phosphate binders (SFO vs. SEV), although SFO resulted in fewer constipated participants. Future interventional studies with a larger sample size may include factors beyond bowel habits and their role in affecting the UTOX levels. 


\section{Acknowledgments}

We would like to thank Nancy Ginsberg, Sujata M. Patel, Bryan T. Stengel, Marcella Modugno, Shannah L. Johnson, Brieana Beach, Caroline M. Williams, and Dr. Jochen Raimann for their contribution to the study; we also thank Dr. John Daugirdas, University of Illinois at Chicago, for initial discussions of the hypothesis. Part of the study has previously been published as an abstract at the 2019 American Society of Nephrology Annual Meeting. The title of the presented poster was "Relationship between Phosphate Binder Type and Gut Microbiome-Derived Uremic Toxin Levels in Hemodialysis Patients."

\section{Statement of Ethics}

All study procedures were approved by Western Institutional Review Board ${ }^{\circledR}$ (Protocol number: \#20181972) and performed in accordance with the principles of the Declaration of Helsinki. Signed written informed consent forms were obtained from all participants for this study.

\section{Conflict of Interest Statement}

P.K. holds stocks and S.T. holds performance shares in Fresenius Medical Care. The other authors declare no competing interest.

\section{Funding Sources}

The study received financial support from the Renal Research Institute.

\section{Author Contributions}

L.W. and L.M.T.S. contributed equally to this work. L.W., L.M.T.S., X.T., J.C., O.T., S.T., P.K., and N.G. contributed to the design and implementation of the research. L.W., L.M.T.S., X.T., J.C., H.Z., and N.G. analyzed the data. L.W., L.M.T.S., P.K., and N.G. wrote the manuscript with input from all authors. N.G. directed the project. All authors provided critical feedback and helped shape the research, analysis, and manuscript.

\section{References}

1 Vanholder R, De Smet R, Glorieux G, Argilés A, Baurmeister U, Brunet P, et al. Review on uremic toxins: classification, concentration, and interindividual variability. Kidney Int. 2003 May;63(5):1934-43.

2 Vanholder R, Fouque D, Glorieux G, Heine GH, Kanbay M, Mallamaci F, et al. Clinical management of the uraemic syndrome in chronic kidney disease. Lancet Diabetes Endocrinol. 2016 Apr;4(4):360-73.

3 Kikuchi K, Itoh Y, Tateoka R, Ezawa A, Murakami K, Niwa T. Metabolomic search for uremic toxins as indicators of the effect of an oral sorbent AST-120 by liquid chromatography/tandem mass spectrometry. J Chromatogr B Analyt Technol Biomed Life Sci. 2010 Nov 1;878(29):2997-3002.

4 Meyer TW, Hostetter TH. Uremic solutes from colon microbes. Kidney Int. 2012 May; 81(10):949-54.

5 Poesen R, Meijers B, Evenepoel P. The colon: an overlooked site for therapeutics in dialysis patients. Semin Dial. 2013 May-Jun;26(3): 323-32.

6 Vaziri ND, Wong J, Pahl M, Piceno YM, Yuan J, DeSantis TZ, et al. Chronic kidney disease alters intestinal microbial flora. Kidney Int. 2013;83(2):308-15.

7 Ramezani A, Massy ZA, Meijers B, Evenepoel $\mathrm{P}$, Vanholder R, Raj DS. Role of the gut microbiome in uremia: a potential therapeutic target. Am J Kidney Dis. 2016 Mar;67(3):48398.

8 Wong J, Piceno YM, DeSantis TZ, Pahl M, Andersen GL, Vaziri ND. Expansion of urease- and uricase-containing, indole- and pcresol-forming and contraction of shortchain fatty acid-producing intestinal micro- biota in ESRD. Am J Nephrol. 2014;39(3): 230-7.

9 Lesaffer G, De Smet R, Lameire N, Dhondt A, Duym P, Vanholder R. Intradialytic removal of protein-bound uraemic toxins: role of solute characteristics and of dialyser membrane. Nephrol Dial Transplant. 2000 Jan;15(1):507.

10 Marquez IO, Tambra S, Luo FY, Li Y, Plummer NS, Hostetter TH, et al. Contribution of residual function to removal of proteinbound solutes in hemodialysis. Clin J Am Soc Nephrol. 2011 Feb;6(2):290-6.

11 Watanabe H, Miyamoto Y, Honda D, Tanaka $\mathrm{H}$, Wu Q, Endo M, et al. p-Cresyl sulfate causes renal tubular cell damage by inducing oxidative stress by activation of NADPH oxidase. Kidney Int. 2013 Apr;83(4):582-92.

12 Vanholder R, Schepers E, Pletinck A, Nagler EV, Glorieux G. The uremic toxicity of indoxyl sulfate and p-cresyl sulfate: a systematic review. J Am Soc Nephrol. 2014 Sep;25(9): 1897-907.

13 Vanholder R, Pletinck A, Schepers E, Glorieux $\mathrm{G}$. Biochemical and clinical impact of organic uremic retention solutes: a comprehensive update. Toxins. 2018 Jan 8;10(1):33.

14 Ikee R, Sasaki N, Yasuda T, Fukazawa S. Chronic kidney disease, gut dysbiosis, and constipation: a burdensome triplet. Microorganisms. 2020 Nov 25;8(12): 1862.

15 Floege J, Covic AC, Ketteler M, Rastogi A, Chong EM, Gaillard S, et al. A phase III study of the efficacy and safety of a novel iron-based phosphate binder in dialysis patients. Kidney Int. 2014 Sep;86(3):638-47.

16 Floege J, Covic AC, Ketteler M, Mann JF, Rastogi A, Spinowitz B, et al. Long-term effects of the iron-based phosphate binder, sucroferric oxyhydroxide, in dialysis patients. Nephrol Dial Transplant. 2015 Jun;30(6): 1037-46.

17 Koiwa F, Yokoyama K, Fukagawa M, Terao A, Akizawa T. Efficacy and safety of sucroferric oxyhydroxide compared with sevelamer hydrochloride in Japanese haemodialysis patients with hyperphosphataemia: a randomized, open-label, multicentre, 12-week phase III study. Nephrology. 2017 Apr;22(4):293300 .

18 Evenepoel P, Meijers BK, Bammens BR, Verbeke $\mathrm{K}$. Uremic toxins originating from $\mathrm{CO}^{-}$ lonic microbial metabolism. Kidney Int Suppl. 2009 Dec(114):S12-9.

19 Sabatino A, Regolisti G, Brusasco I, Cabassi A, Morabito S, Fiaccadori E. Alterations of intestinal barrier and microbiota in chronic kidney disease. Nephrol Dial Transplant. 2015 Jun; 30(6):924-33.

20 Lewis SJ, Heaton KW. Stool form scale as a useful guide to intestinal transit time. Scand J Gastroenterol. 1997 Sep;32(9):920-4.

21 Dunn WB, Broadhurst D, Begley P, Zelena E, Francis-McIntyre S, Anderson N, et al. Procedures for large-scale metabolic profiling of serum and plasma using gas chromatography and liquid chromatography coupled to mass spectrometry. Nat Protoc. 2011 Jun 30;6(7): 1060-83.

$22 \mathrm{Wu}$ MJ, Chang CS, Cheng $\mathrm{CH}$, Chen $\mathrm{CH}$, Lee WC, Hsu YH, et al. Colonic transit time in long-term dialysis patients. Am J Kidney Dis. 2004 Aug;44(2):322-7.

23 Tanaka H, Sirich TL, Meyer TW. Uremic solutes produced by colon microbes. Blood $\mathrm{Pu}$ rif. 2015;40(4):306-11. 
24 Roager HM, Hansen LB, Bahl MI, Frandsen HL, Carvalho V, Gøbel RJ, et al. Colonic transit time is related to bacterial metabolism and mucosal turnover in the gut. Nat Microbiol. 2016 Jun 27;1(9):16093.

25 Wing MR, Patel SS, Ramezani A, Raj DS. Gut microbiome in chronic kidney disease. Exp Physiol. 2016 Apr;101(4):471-7.

26 Ramos CI, Armani RG, Canziani ME, Ribeiro Dolenga CJ, Nakao LS, Campbell KL, et al. Bowel habits and the association with uremic toxins in non-dialysis-dependent chronic kidney disease patients. J Ren Nutr. 2020 Jan; 30(1):31-5.

27 Pereira NBF, Ramos CI, de Andrade LS, Teixeira RR, Nakao LS, Rodrigues SD, et al. Influence of bowel habits on gut-derived toxins in peritoneal dialysis patients. J Nephrol. 2020 Oct;33(5):1049-57.

28 Biruete A, Hill Gallant KM, Lindemann SR, Wiese GN, Chen NX, Moe SM. Phosphate binders and nonphosphate effects in the gastrointestinal tract. J Ren Nutr. 2020 Jan;30(1): 4-10.

29 Dai L, Meijers BK, Bammens B, de Loor H, Schurgers LJ, Qureshi AR, et al. Sevelamer use in end-stage kidney disease (ESKD) patients associates with poor Vitamin $\mathrm{K}$ status and high levels of gut-derived uremic toxins: a drug-bug interaction? Toxins. 2020 May 27; 12(6):351
30 Lin CJ, Pan CF, Chuang CK, Liu HL, Huang $\mathrm{SF}$, Chen $\mathrm{HH}$, et al. Effects of sevelamer hydrochloride on uremic toxins serum indoxyl sulfate and p-cresyl sulfate in hemodialysis patients. J Clin Med Res. 2017 Sep;9(9):76570.

31 Riccio E, Sabbatini M, Bruzzese D, Grumetto L, Marchetiello C, Amicone M, et al. Plasma $\mathrm{p}$-cresol lowering effect of sevelamer in nondialysis CKD patients: evidence from a randomized controlled trial. Clin Exp Nephrol. 2018 Jun;22(3):529-38.

32 Brandenburg VM, Schlieper G, Heussen N, Holzmann S, Busch B, Evenepoel P, et al. Serological cardiovascular and mortality risk predictors in dialysis patients receiving sevelamer: a prospective study. Nephrol Dial Transplant. 2010 Aug;25(8):2672-9.

33 Bennis Y, Cluet Y, Titeca-Beauport D, El Esper N, Ureña P, Bodeau S, et al. The effect of sevelamer on serum levels of gut-derived uremic toxins: results from in vitro experiments and a multicenter, double-blind, placebocontrolled, randomized clinical trial. Toxins. 2019 May 17;11(5):279.

34 Lenglet A, Fabresse N, Taupin M, Gomila C, Liabeuf S, Kamel S, et al. Does the administration of sevelamer or nicotinamide modify uremic toxins or endotoxemia in chronic hemodialysis patients? Drugs. 2019 Jun;79(8): 855-62.
35 Iguchi A, Yamamoto S, Oda A, Tanaka K, Kazama JJ, Saeki T, et al. Effect of sucroferric oxyhydroxide on gastrointestinal microbiome and uremic toxins in patients with chronic kidney disease undergoing hemodialysis. Clin Exp Nephrol. 2020 Aug;24(8): 725-33.

36 Park JC, Kovesdy CP, Duong U, Streja E, Rambod M, Nissenson AR, et al. Association of serum alkaline phosphatase and bone mineral density in maintenance hemodialysis patients. Hemodial Int. 2010 Apr;14(2):182-92.

37 Koiwa F, Yokoyama K, Fukagawa M, Akizawa T. Long-term assessment of the safety and efficacy of PA21 (Sucroferric Oxyhydroxide) in Japanese hemodialysis patients with hyperphosphatemia: an open-label, multicenter, Phase III Study. J Ren Nutr. 2017 Sep;27(5): 346-54.

38 Sprague SM, Ketteler M, Covic AC, Floege J, Rakov V, Walpen S, et al. Long-term efficacy and safety of sucroferric oxyhydroxide in African American dialysis patients. Hemodial Int. 2018 Oct;22(4):480-91.

39 Gray K, Ficociello LH, Hunt AE, Mullon C, Brunelli SM. Phosphate binder pill burden, adherence, and serum phosphorus control among hemodialysis patients converting to sucroferric oxyhydroxide. Int J Nephrol Renovasc Dis. 2019;12:1-8. 